\title{
Nonaxisymmetric magnetic field generation in rapidly rotating late-type stars
}

\author{
D. Moss \\ School of Mathematics, University of Manchester, Manchester M13 9PL, UK \\ e-mail: moss@ma.man.ac.uk \\ Received 22 July 2004 / Accepted 31 October 2004

\begin{abstract}
Recently the discovery of the "flip-flop" phenomenon, by which a predominant "active" longitude jumps by about $180^{\circ}$, on several rapidly rotating late-type stars has directed attention to the issues surrounding the presence of large-scale rotation laws, acting in spherical shells. Stable nonaxisymmetric magnetic fields are found to be generated for a range of parameters and, for certain of these models, jumps in a measure plausibly related to the position of active longitudes are found to occur. In general, nonaxisymmetric fields appear to be easier to generate in thinner dynamo-active regions. However, flip-flops appear to be stronger for intermediate thicknesses of the dynamo-active shells.
\end{abstract} \\ nonaxisymmetric magnetic fields in these objects. Here we study nonlinear mean field dynamo models with quasi-cylindrical
}

Key words. magnetic fields - magnetohydrodynamics - stars: activity - stars: late-type - stars: rotation - stars: magnetic fields

\section{Introduction}

Late type stars have been extensively observed in the last decade or so allowing, inter alia, determination of surface differential rotation laws (e.g. Hall 1991; Donahue 1993; Henry et al. 1995) and sometimes their temporal variations (e.g. Collier Cameron \& Donati 2002; Donati et al. 2003; Messina $\&$ Guinan 2003). Both optical photometry and doppler imaging techniques have revealed large-scale nonaxisymmetric surface features on a number of stars that rotate relatively rapidly compared to the Sun. These features are often of lower temperature than their surroundings and, by analogy to sunspots, thought to be the site of strong magnetic fields. In a few cases, this interpretation is supported by direct determination of field strengths. However these features often occupy a much larger fraction of the stellar surface than even the largest sunspots - a striking example is AB Dor (Jardine et al. 2002). More indirectly, active longitudes, again inferentially associated with nonaxisymmetric magnetic structures, are frequently detected (e.g. Hall 1991; Jetsu 1996). Thus, if the source of the magnetic field is a dynamo operating in (or just below) the sub-surface convection zone, then excitation and maintenance of large-scale magnetic fields that are nonaxisymmetric with respect to the rotation axis seems to be implied.

Nonlinear stellar dynamo models with stable large-scale nonaxisymmetric fields are scarce in the otherwise voluminous astrophysical dynamo literature. The earliest results appear to be due to Rädler et al. (1990) and Moss et al. (1991). They agreed that when an algebraic alpha-quenching is the only nonlinearity, then with rotation laws of the form $\Omega=\Omega(r)$ rather artificial distributions of $\Omega$ and the dynamo coefficient $\alpha$ are required to maintain nonaxisymmetric fields. Later, Barker \& Moss (1994) and Moss et al. (1995) studied models in which the large-scale Lorentz force acting on, and so modifying, the underlying rotational velocity field $\Omega_{0}(r)$ was the sole nonlinearity. They found that stable nonaxisymmetric fields were now more readily obtained, without prior tuning of the spatial dependence of $\alpha$ and $\Omega_{0}$. The latitudinal dependence of the resulting angular velocity seems to be an important feature. An analytic study by Bassom et al. (2005) has also demonstrated the importance of latitudinal differential rotation in this context. In some instances, a dynamically consistently meridional flow appeared also to play a role. In all of these studies the final stable state was either purely axisymmetric, or had no axisymmetric part.

More recently, Moss (1999) studied an alpha-quenched solar model, using a rotation law derived from helioseismic observations, and found that a weak nonaxisymmetric field could coexist with a dominant axiymmetric field. This result has possible applications to the solar "sector structure", and appears to be the first published example of a stable model field configuration containing both axisymmetric and nonaxisymmetric parts.

Jetsu et al. (1991) found the predominant active longitude of the rapidly rotating giant FK Comae to have changed suddenly by $180^{\circ}$. This was the first reported instance of the "flipflop" phenomenon, which has subsequently been found to recur on FK Comae (Korhonen et al. 2001, 2002), and also reported to be present on other late-type stars. These include the single young active dwarf LQ Hydrae (Berdyugina et al. 2002), and even the Sun - Berdyugina \& Usoskin (2003) - as well as 
certain RS CVn binary systems (e.g. Berdyugina \& Tuominen 1998). A preliminary account of the first dynamo model to address this intriguing behaviour was presented by Moss (2004).

Thus, the presence of large-scale nonaxisymmetric magnetic fields at the surfaces of late-type stars, both single and binary, appears to be a widespread and challenging phenomenon. Close binaries are intrinsically nonaxisymmetric, and this may directly influence the nature of their fields (see Moss \& Tuominen 1997; Moss et al. 2002), as well as promoting rapid rotation of the component stars. Such effects of binarity will not be considered explicitly here.

In this paper a survey is made of alpha-quenched mean field dynamo models, in spherical shells of varying thickness corresponding in a very approximate way to stars of different spectral types. The rapid rotation is modelled by adopting a quasicylindrical rotation law, as is often thought to be appropriate for rapid rotators. Taking $\Omega \propto \operatorname{constant}\left(1+b \sin ^{2} \theta\right)$, with $b$ a small constant, at the surface is consistent with the available observational evidence. Of course, the dynamo model used here is grossly simplified, and can be criticized on a number of grounds. However it is reasonable to use such a model to make a preliminary study of a hitherto unaddressed problem.

\section{The model}

The standard alpha-quenched mean field dynamo equation

$$
\frac{\partial \boldsymbol{B}}{\partial t}=\nabla \times\left((\Omega \times \boldsymbol{r}) \times \boldsymbol{B}+\frac{\alpha_{0}(r, \theta)}{\left(1+\boldsymbol{B}^{2} / B_{\mathrm{eq}}^{2}\right)}-\eta \nabla \times \boldsymbol{B}\right),
$$

is solved by the method described in Moss (2004) - see Moss et al. (1991) for a fuller account. $r, \theta, \lambda$ are spherical polar coordinates, and the field is represented as the sum of modes $\propto \exp (i m \lambda), m=1, \ldots M . B_{\text {eq }}$ is a uniform reference field strength, conventionally that of an equipartition field. The simplest assumption, $\alpha_{0}=\alpha_{*} \cos \theta$ with $\alpha_{*}=$ const, was made for the alpha-coefficient, and the turbulent diffusivity $\eta$ was taken to be uniform. In order to capture the essence of a rapidly rotating spherical shell,

$\Omega / \Omega_{0}=1+0.5\left(1+\operatorname{erf}\left(\frac{\left(r-r_{\mathrm{c}}\right)}{d}\right)\right) a r^{2} \sin ^{2} \theta$

where $\Omega_{0}, a, r_{\mathrm{c}}$ and $d$ are constants. The computational domain extends over $r_{0} \leq r \leq R$, and $\Omega$ thus undergoes a transition between approximately solid body rotation at $r=r_{0}$ to a cylindrical law in $r>r_{\mathrm{c}}$, through a transition zone of halfwidth $d$ centred at $r=r_{\mathrm{c}}$. Contours $\Omega=$ constant are shown in Fig. 1 when $r_{0}=0.64, r_{\mathrm{c}}=0.70, d=0.05-$ see Sect. 3.1. Nondimensionalization in terms of the length $R$, time $R^{2} / \eta$ and equipartition field $B_{\text {eq }}$ yields the standard dynamo parameters

$C_{\alpha}=\frac{\alpha_{*} R}{\eta}, \quad C_{\omega}=\frac{\Omega_{0} R^{2}}{\eta}$.

However the crucial parameter for dynamo action is

$C_{\omega}^{*}=a C_{\omega}$,

which measures the strength of the differential rotation. From here onwards, all variables are in dimensionless form, unless

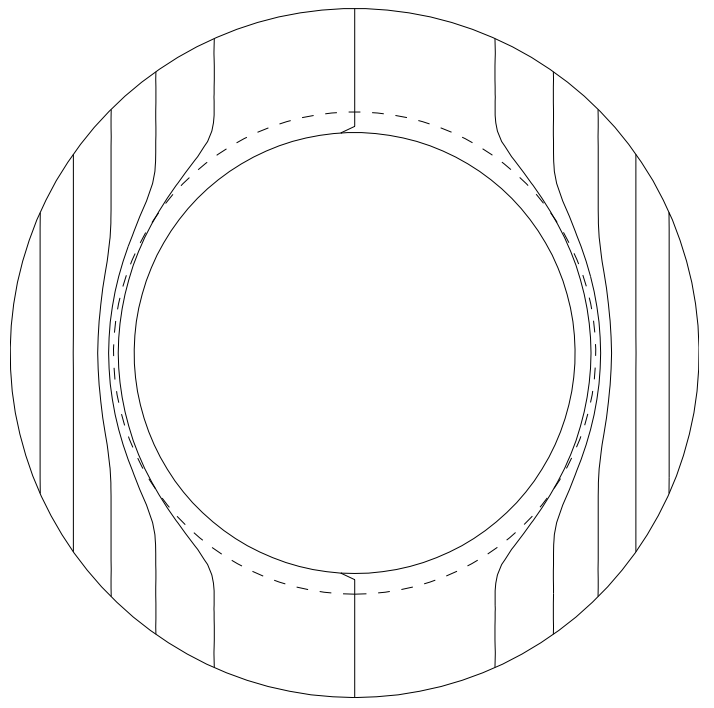

Fig. 1. Contours of $\Omega=$ const when $r_{0}=0.64$. The broken circle is $r=r_{\mathrm{c}}=0.70$.

otherwise stated. Note that this is an " $\alpha$ ' $\omega$ " dynamo model the alpha term is kept in both the poloidal and toroidal parts of Eq. (1).

For some orientation, note that if $P_{\text {rot }} \sim 0.5 \mathrm{~d}, R \sim R_{\odot}$, $a \sim 0.007$ (cf. AB Doradus) then with $\eta_{0}=10^{12} \mathrm{~cm}^{2} \mathrm{~s}^{-1}$, $C_{\omega}^{*} \approx 500 . C_{\alpha}$ is more difficult to estimate, but values $10 \lesssim$ $\left|C_{\alpha}\right| \lesssim 100$ seem acceptable.

A standard meridional grid of 101 points uniformly distributed over $0 \leq \theta \leq \pi$ and 61 points over $r_{0} \leq r \leq 1$ was used. Preliminary solutions were computed with $M=1$, and selected solutions recomputed with $M=3$. Except for a number of cases where the fields were steady, the solutions with $M=3$ agreed well with those with $M=1-$ the energy in modes $m>1$ decreases rapidly (see also Moss et al. 1991). At $r=1$ the solutions were matched to a vacuum solution in $r>1$; this is a plausible choice, but somewhat arbitrary. (For example, the field above the solar surface is certainly not a vacuum field.) For the solutions discussed explicitly below, the boundary conditions at $r=r_{0}$ are that the radial component of the poloidal field and the toroidal field are each zero there. An experiment with "overshoot" boundary conditions, modelling the field becoming zero a small distance below this boundary, gave very similar results (cf. Moss et al. 1995; Moss 1999). Of course, other choices of boundary conditions here are possible, and it cannot be ruled out a priori that they do not lead to somewhat different behaviour.

A gross characterisation of the solutions is provided by the global measure $\mathcal{M}=E_{\text {nax }} / E_{\text {tot }}$, where $E_{\text {tot }}=E_{\text {nax }}+E_{\text {ax }}$ is the sum of the energies in the nonaxisymmetric and axisymmetric parts of the magnetic field, taken through the entire dynamo active region, $r_{0} \leq r \leq 1$. It is at least arguable that the nature of the observable/inferred fields could be better represented by the value of $\mathcal{M}$ calculated over a relatively shallow subsurface shell. However, such calculations did not give significantly different values, and so only the global value of $\mathcal{M}$ is discussed. Other global measures are $P_{m}=\left(E_{m}^{\mathrm{e}}-E_{m}^{\mathrm{o}}\right) / E_{m}$, the parity with respect to the rotational equator of the part of the 
Table 1. The parameters of the underlying "stellar" models.

\begin{tabular}{ccc}
\hline \hline$r_{0}$ & $r_{\mathrm{c}}$ & $d$ \\
\hline 0.20 & 0.25 & 0.05 \\
0.64 & 0.70 & 0.05 \\
0.77 & 0.80 & 0.05 \\
\hline
\end{tabular}

magnetic field $\propto \exp (\operatorname{im} \lambda)$, and $\mathcal{P}$ the overall field parity (see Moss et al. 1991). (Here $E_{m}^{\mathrm{e}}, E_{m}^{\mathrm{o}}$ are the energies in the $m$ th mode with even and odd symmetry respectively with respect to the rotational equator.)

\section{Results}

Three models were considered, as specified in Table 1. The choice of parameters is somewhat arbitrary, but designed to illustrate the effects of moving from a shallow to a deep convective envelope. It might have been reasonable to have reduced somewhat the value of $d$ when $r_{0}=0.77$ and $r_{\mathrm{c}}-r_{0}$ is smaller than the other cases, but this was found to cause numerical inconvenience.

The nominal value $C_{\omega}=10^{4}$ was taken throughout. $a$ was varied within the range $+1.0 \lesssim a \lesssim-1.0$, but extreme values in this range were not systematically investigated; thus $-10^{4} \lessgtr C_{\omega}^{*} \lessgtr 10^{4}$. When $a>0\left(C_{\omega}^{*}>0\right)$ the surface rotation is "solar-like" with equatorial acceleration, and correspondingly $a<0$ corresponds to "anti-solar" surface behaviour. The current evidence is that solar-like surface behaviour is probably the norm, but there have also been reports of anti-solar behaviour for a small number of stars (e.g. Messina \& Guinan 2003). For the broader investigation, only negative values of $C_{\alpha}$ were considered, consistent with simple mean field solar models using a modern rotation law that reproduce the correct sense of field migration. The case $r_{0}=0.64$ was studied more closely, and here some computations were also made with $C_{\alpha}>0$.

The results presented are not exhaustive: the computations are fairly lengthy, and were started either from an arbitrary initial state or sometimes, when $M=3$, continued from the corresponding (or a similar) solution with $M=1$. No systematic search for the existence of multiple stable solutions for given parameters was made, although these are known to exist for $\alpha^{2} \omega$ dynamos in spherical geometry (e.g. Brandenburg et al. 1989; Moss et al. 1995). By chance, stable axisymmetric solutions of odd and even parity were found to coexist for certain combinations of parameters where the field configuration is reported below as "axisymmetric", i.e. $\mathcal{M}=0$. In some instances, one of these solutions was steady, the other oscillatory. For this reason, no attempt to classify stable axisymmetric solutions by their parity was made, and in these cases it may be that where steady solutions are indicated by asterisks in Tables 2-4, oscillatory solutions can also be found. Solutions containing a mixture of axisymmetric and nonaxisymmetric fields may have either steady or oscillating values of the $P_{i}$; there then appears to be a pattern to the distribution of the values of the $P_{i}-$ see below. It is not precluded that stable mixed axisymmetric and nonaxisymmetric solutions might be found to coexist for certain choices of parameters, but such cases were not found here.

\subsection{Models with dynamo region of intermediate thickness: $r_{0}=0.64$}

Gross properties of these solutions are summarized in Table 2. $\mathcal{M}$ gives the degree of nonaxisymmetry, with $\mathcal{M}=0$ corresponding to purely axisymmetric solutions, $\mathcal{M}=1$ to solutions with no axisymmetric part, and intermediate values indicating solutions with both axisymmetric and nonaxisymmetric parts. Asterisks indicate that only steady solutions were found, although as noted above this does not mean that other solutions do not exist. For orientation, when $C_{\omega}^{*}=10^{3}$, marginal values of $C_{\alpha}$ when $C_{\alpha}<0$ are approximately -7.5 and -14.3 for the $m=0$ and $m=1$ modes respectively. When $C_{\omega}^{*}=500$, the corresponding values are -11.6 and -13.4 , whereas for $C_{\omega}^{*}=-500$ the marginal values for modes $m=0,1$ are $C_{\alpha, \mathrm{c}}=-11.9$ and -12.6 respectively. Unsurprisingly, for large enough $C_{\omega}^{*}$ only axisymmetric solutions are found - strong radial differential rotation is known preferentially to enhance decay of nonaxisymmetric fields. Similarly, for large enough $\left|C_{\alpha} / C_{\omega}^{*}\right|$, solutions are often steady, approaching the $\alpha^{2}$ regime - see also Tables 3 and 4 . However there is a distinct asymmetry between behaviours with $C_{\omega}^{*}>0$ and $C_{\omega}^{*}<0$, with nonaxisymmetric solutions being more readily found in the latter case; this situation is at least hinted at by the smaller difference between the marginal $C_{\alpha}$ values in the latter case - see above. The solution for $C_{\alpha}=-100, C_{\omega}^{*}=500$ is discussed in detail in Moss (2004).

Figure 2 shows the variations of energies and parities with time for $C_{\alpha}=-50, C_{\omega}^{*}=500$ and $C_{\alpha}=-50, C_{\omega}^{*}=-500$; and snapshots of the surface magnetic field configurations for these solutions are shown in Fig. 3. These are quite typical of nonsteady mixed mode solutions. Remarkably, in all the cases investigated for this model, and for all other values of $r_{0}$, a simple dichotomy was found: when $C_{\alpha} C \omega^{*}>0, P_{0} \rightarrow+1, P_{1} \rightarrow-1$ whereas for $C_{\alpha} C \omega^{*}<0, P_{0}$ oscillates quite widely and $P_{1}$ has small variations near 0.0 - see, e.g., Figs. 2a,b. (Given the extended transient behaviour sometimes present in solutions with $C_{\alpha} C \omega^{*}>0$, it cannot always be ruled out that, in the final stable state, $P_{1}$ is very near to -1 rather than $P_{1}=-1$ exactly.)

\subsection{Models with deep dynamo region: $r_{0}=0.20$}

Here, with $C_{\omega}^{*}=10^{3}$ and $C_{\alpha}<0$, marginal dynamo numbers $C_{\alpha}$ for the $m=0$ and $m=1$ modes are approximately -5.5 and -12.7 respectively. Results are summarized in Table 3.

\subsection{Models with shallow dynamo region: $r_{0}=0.77$}

Now for $C_{\omega}^{*}=1000$, marginal values of $C_{\alpha}<0$ are approximately -15 and -18 for modes $m=0,1$ respectively, and for $C_{\omega}^{*}=500$ they are little changed at approximately -15 and -17 . A summary of results is given in Table 4, and Fig. 5 shows the time variation of energies and parities for solutions with $C_{\alpha}=-50, C_{\omega}^{*}= \pm 500$. Snapshots of the surface magnetic field distribution are given in Fig. 4. 

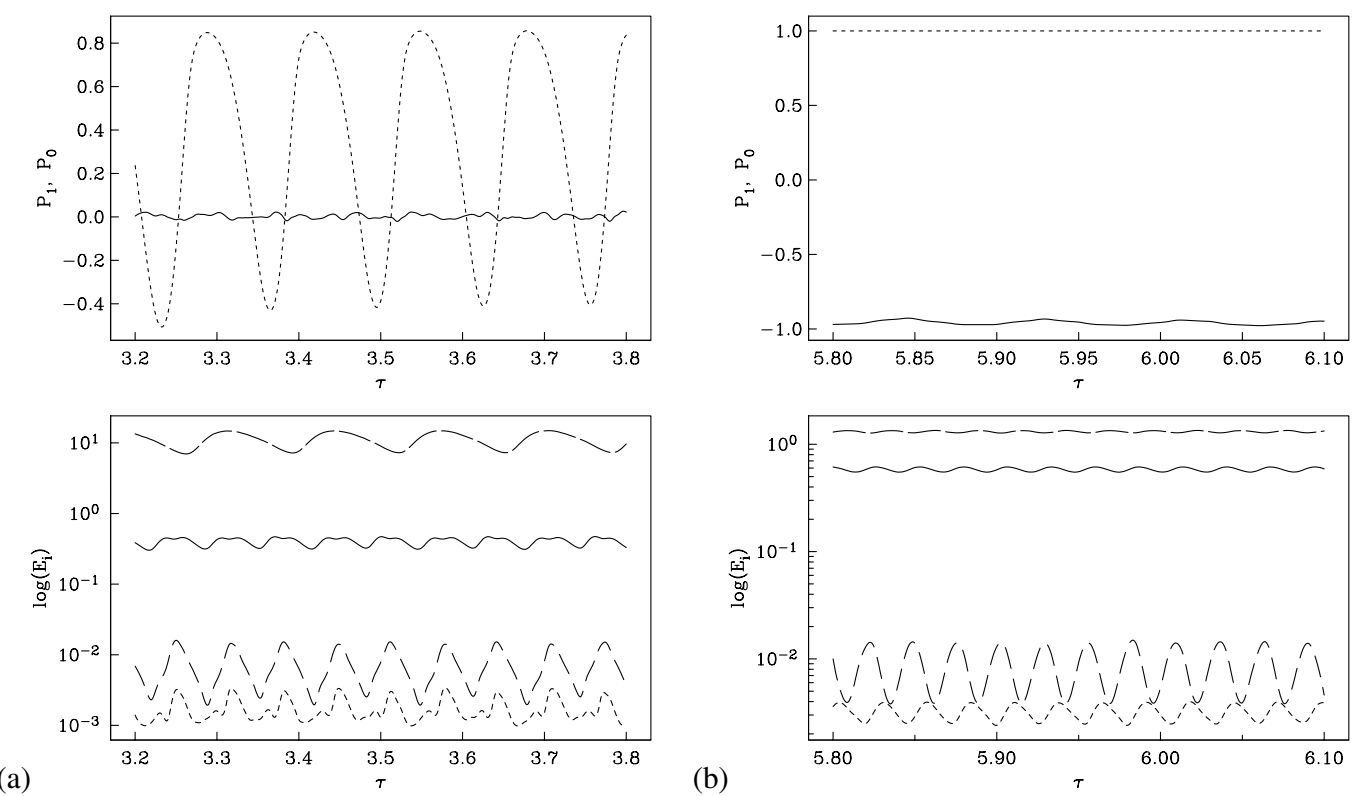

Fig. 2. For $r_{0}=0.64$, variations of parity (top: solid for $P_{1}$, broken for $P_{0}$ ) and modal energies (bottom: $E_{0}$ is upper long-dashed, $E_{1}$ is solid, $E_{2}$ is lower long-dashed, $E_{4}$ short-dashed) for $r_{0}=0.64, C_{\alpha}=-50$ and a) $C_{\omega}^{*}=+500$, b) $C_{\omega}^{*}=-500$. In $\left.\mathbf{b}\right)$ a long transient is decaying as $P_{1} \rightarrow-1$.

Table 2. Summary of results for models with $r_{0}=0.64 . \mathcal{M}$ is the measure of the mean nonaxisymmetry of the fields, with $\mathcal{M}=0,1$ corresponding to purely axisymmetric solutions and solutions with no axisymmetric part, respectively. Asterisks indicate steady solutions.

\begin{tabular}{rrrrrrrrrr}
\hline \hline$C_{\alpha}$ & 100 & 50 & 20 & -17.5 & -20 & -25 & -50 & -100 & -125 \\
\hline$C_{\omega}^{*}$ & & & & & & & & & \\
\hline 4000 & & & & & 0.0 & & 0.0 & & \\
\hline 2000 & & & & & 0.0 & & 0.0 & & \\
\hline 1000 & & 0.11 & & & 0.0 & & $0.0^{*}$ & $0.0^{*}$ & $0.0^{*}$ \\
\hline 500 & 0.29 & 0.31 & 0.45 & $0.0^{*}$ & $0.0^{*}$ & $0.0^{*}$ & 0.03 & 0.06 & 0.05 \\
\hline 100 & & & & $0.0^{*}$ & $0.0^{*}$ & $0.0^{*}$ & $0.0^{*}$ & $0.0^{*}$ & $0.0^{*}$ \\
\hline-100 & & & & $1.0^{*}$ & & & $1.0^{*}$ & & \\
\hline-500 & 0.03 & 0.0 & 0.00 & 0.58 & & 0.36 & 0.30 & 0.30 & \\
\hline-1000 & & 0.0 & & 0.35 & & 0.07 & 0.11 & 0.14 & \\
\hline-2000 & & & & & & 0.01 & 0.04 & 0.06 & \\
\hline-5000 & & & & & & 0.0 & & & \\
\hline
\end{tabular}

\section{Active longitudes and the flip-flop phenomenon}

\subsection{General considerations}

The presence of large-scale nonaxisymmetric fields at a stellar surface means that observed measures of field strength vary with the phase of observation (if not observed from above the rotational poles), in addition to any intrinsic temporal variations. If stellar surface activity is associated with field strength and not sign, then purely nonaxisymmetric fields with dominant $m=1$ azimuthal dependence can be expected to display active longitudes (two in the simplest case), whatever their parity. Moreover, given that nonaxisymmetric structures in general rotate rigidly with respect to the equatorial surface rotation, then active longitudes will do likewise.
The situation is rather more complex when both axisymmetric and nonaxisymmetric fields are present (i.e. $\mathcal{M} \neq 0,1$ ). Exactly what would be observed depends on a number of factors, including the geometries and relative strengths of both the $m=0$ and $m=1$ parts of the field, and the inclination of the rotation axis to the line of sight. For example, an $\mathrm{S} 1$ field ("perpendicular dipole-like", $P_{1}=+1$ ) will have positive radial component in one hemisphere over all latitudes (Fig. 6a), whereas an A1 field $\left(P_{1}=-1\right)$, illustrated in cross-section in Fig. 6b, will exhibit a predominantly positive radial component for half a rotation period when viewed from inclination $i \approx 45^{\circ}$, but will show equal positive and negative contributions from $i \approx 90^{\circ}$.

In the computed models, these fields are usually combined with an oscillatory axisymmetric field. Figure 7 shows 


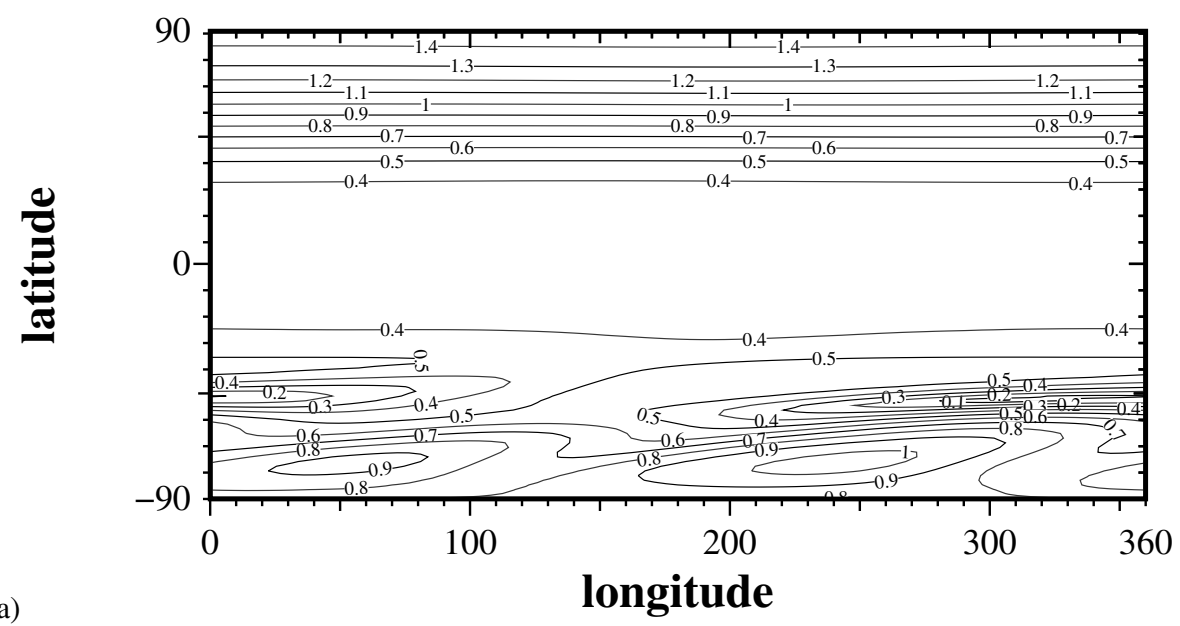

(a)

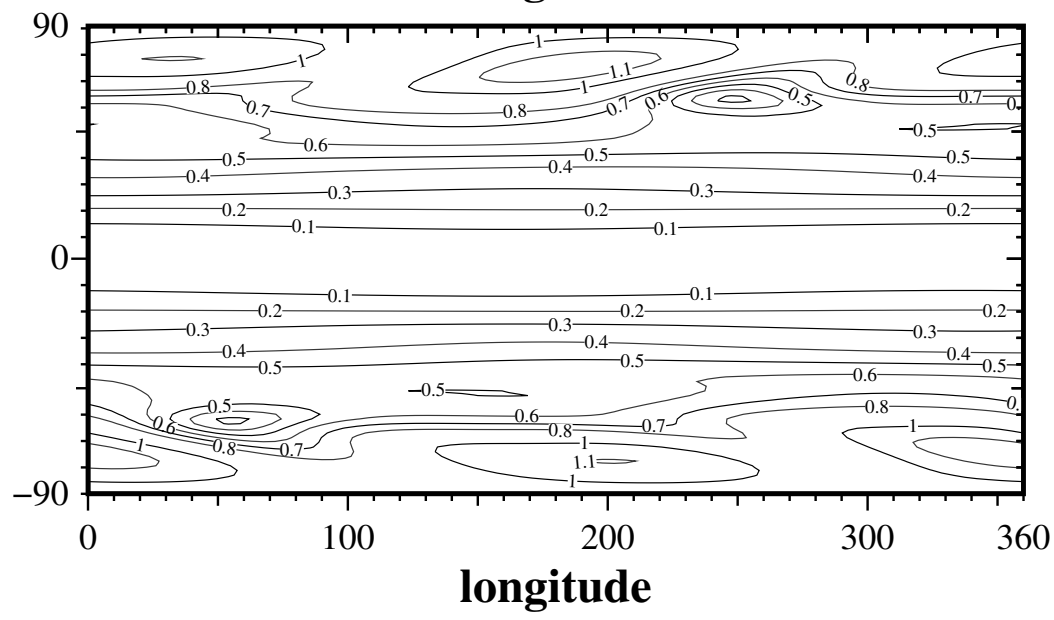

Fig. 3. Snapshot of contours of surface field strength for $r_{0}=0.64, C_{\alpha}=-50$ and a) $C_{\omega}^{*}=+500$, b) $C_{\omega}^{*}=-500$. Panel b) is for the stable configuration with $P_{0}=P_{2}=+1, P_{1}=P_{3}=-1$, after the transient shown in Fig. 2 has disappeared.

Table 3. Summary of results for models with $r_{0}=0.20 . \mathcal{M}$ is the measure of the mean nonaxisymmetry of the fields, with $\mathcal{M}=0,1$ corresponding to purely axisymmetric solutions and solutions with no axisymmetric part, respectively. Asterisks indicate steady solutions, and $U$ that a numerical instability was encountered.

\begin{tabular}{rcccc}
\hline \hline$C_{\alpha}$ & -15 & -25 & -50 & -100 \\
\hline$C_{\omega}^{*}$ & & & \\
\hline 2000 & & 0.0 & $0.0^{*}$ & \\
\hline 1000 & & & 0.0 & $0.0^{*}$ \\
\hline 500 & 0.0 & 0.0 & 0.0 & $0.0^{*}$ \\
\hline 200 & $0.0^{*}$ & 0.0 & 0.0 & $0.0^{*}$ \\
\hline-200 & & $1.0^{*}$ & $\mathrm{U}$ & \\
\hline-500 & & 0.15 & 0.24 & 0.0 \\
\hline-1000 & & 0.025 & 0.0 & $\mathrm{U}$ \\
\hline-2000 & & 0.0 & $10^{-7}$ & $\mathrm{U}$ \\
\hline
\end{tabular}

a cross-section of an S1 field (concentrated somewhat to higher latitudes) combined with an S0 field ("aligned quadrupolelike"). It is clear that seen from large inclinations $i$ in the plane of the figure, the fields reinforce over much of one hemisphere, and cancel over the other. Thus there would be one dominant
Table 4. Summary of results for models with $r_{0}=0.77 . \mathcal{M}$ is the measure of the mean nonaxisymmetry of the fields, with $\mathcal{M}=0,1$ corresponding to purely axisymmetric solutions and solutions with no axisymmetric part, respectively. Asterisks indicate steady solutions.

\begin{tabular}{|c|c|c|c|c|c|}
\hline$\overline{C_{\alpha}}$ & -20 & -30 & -50 & -100 & -200 \\
\hline \multicolumn{6}{|l|}{$C_{\omega}^{*}$} \\
\hline 4000 & & & $0.0^{*}$ & & \\
\hline 2000 & & $0.0^{*}$ & $0.0^{*}$ & & \\
\hline 1000 & $0.0^{*}$ & & 0.03 & 0.03 & $0.0^{*}$ \\
\hline 500 & $0.0^{*}$ & $0.0^{*}$ & 0.16 & $0.0^{*}$ & \\
\hline 200 & $0.0^{*}$ & & $0.0^{*}$ & & $0.33^{*}$ \\
\hline-200 & $1.0^{*}$ & & $1.0^{*}$ & & $1.0^{*}$ \\
\hline-500 & $0.0^{*}$ & & 0.67 & & 0.49 \\
\hline-1000 & $1.0^{*}$ & & 0.37 & & 0.37 \\
\hline-2000 & & & 0.57 & & \\
\hline-4000 & & & 0.03 & & \\
\hline-8000 & & & 0.01 & & \\
\hline
\end{tabular}

active longitude. If the S1 field is of constant sign and the S0 field changes sign as it oscillates regularly, then the active longitude will switch by $180^{\circ}$ (in the frame rotating with the S1 


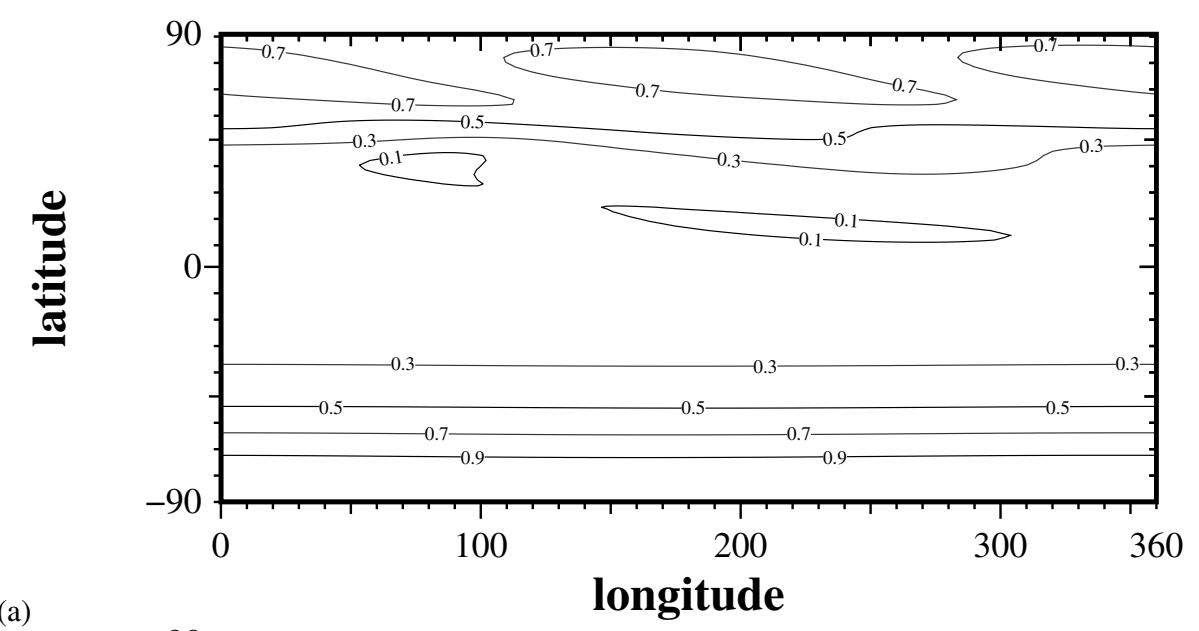

(a)

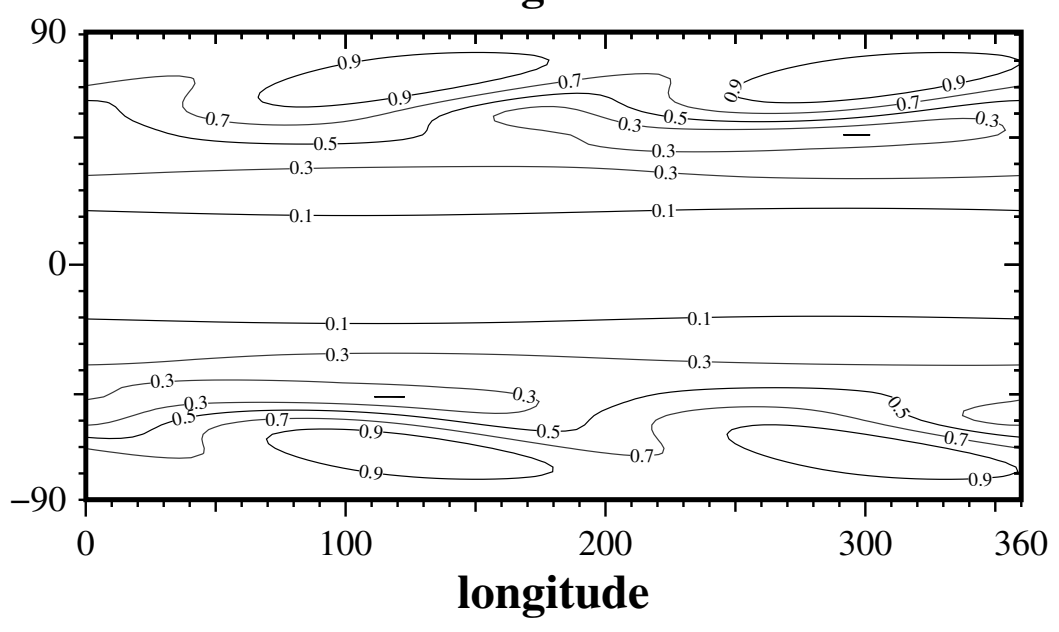

(b)

Fig. 4. Snapshot of contours of surface field strength for $r_{0}=0.77, C_{\alpha}=-50$ and a) $C_{\omega}^{*}=+500$, b) $C_{\omega}^{*}=-500$.

field) every half cycle. A similar argument can be presented for an oscillating A0 field combined with a steady A1 field, or even with a steady $P_{1}=0(\mathrm{~A} 1+\mathrm{S} 1)$ field.

Now suppose that the S0 field in Fig. 7 is replaced by an A0 field. Then over one longitudinal hemisphere there will be cancellation in the northern hemisphere and addition over the southern. Thus measures of integrated field strength taken along a complete meridian will be the same at opposite longitudes and so, from inclinations of near $90^{\circ}$, there will not be a single dominant longitude. However viewed from small inclinations ( $a y i 4^{\circ}$ ) so that a single hemisphere ( $\mathrm{N}$ or $\mathrm{S}$ ) dominates, the situation is rather different. These issues are also discussed by Fluri \& Berdyugina (2005).

For the models with $C_{\alpha} C_{\omega}^{*}>0$ described in Sect. 3, the field components are not of "pure" parity, i.e. $P_{i} \neq \pm 1$. Further, it is known from studies of axisymmetric dynamos in thin shells that the preferred parity can depend quite sensitively on details of how the model is set up. Although this was not investigated here, this result may hold for fields with a nonaxisymmetric part. Thus predicting whether "flip-flops" occur in a given dynamo model, and how they would appear to a distant observer, is a rather uncertain business. In general, a suitable state for observable flip-flops to occur would seem to be one in which the axisymmetric field oscillates, changing sign in the cycle, with neither the axisymmetric nor the nonaxisymmetric component too much stronger than the other (but note that Fluri \& Berdyugina 2005, discuss a flip-flop mechanism where none of the field modes change sign during a cycle). Given that the exact mechanism for formation of active regions is unknown and also that the threshold field strength difference for a predominant active longitude to be recognized is also uncertain, it is difficult to be more definite. The results presented in Tables 2-4 must be viewed in this light.

\subsection{Specific cases}

Here we consider in detail some of the computations tabulated above. The results are presented in two ways.

At a given time, the square of the modulus of the magnetic field at the immediately sub-surface radial mesh point is integrated from pole to pole at a range of longitudes $\phi$, yielding $\overline{B^{2}(\phi)}$. From this the longitude, $\phi_{m}$, with maximum $\overline{B^{2}(\phi)}$ can be found. For any computation, a time series of $\phi_{m}(t)$ can be constructed. Nonaxisymmetric field structures will generally rotate (with respect to the background angular velocity $\Omega_{0}$ ), so $\phi_{m}$ for a steady, purely nonaxisymmetric, field can be expected to change monotonically with time. Here, we have in general a combination of a (weakly) time dependent nonaxisymmetric 

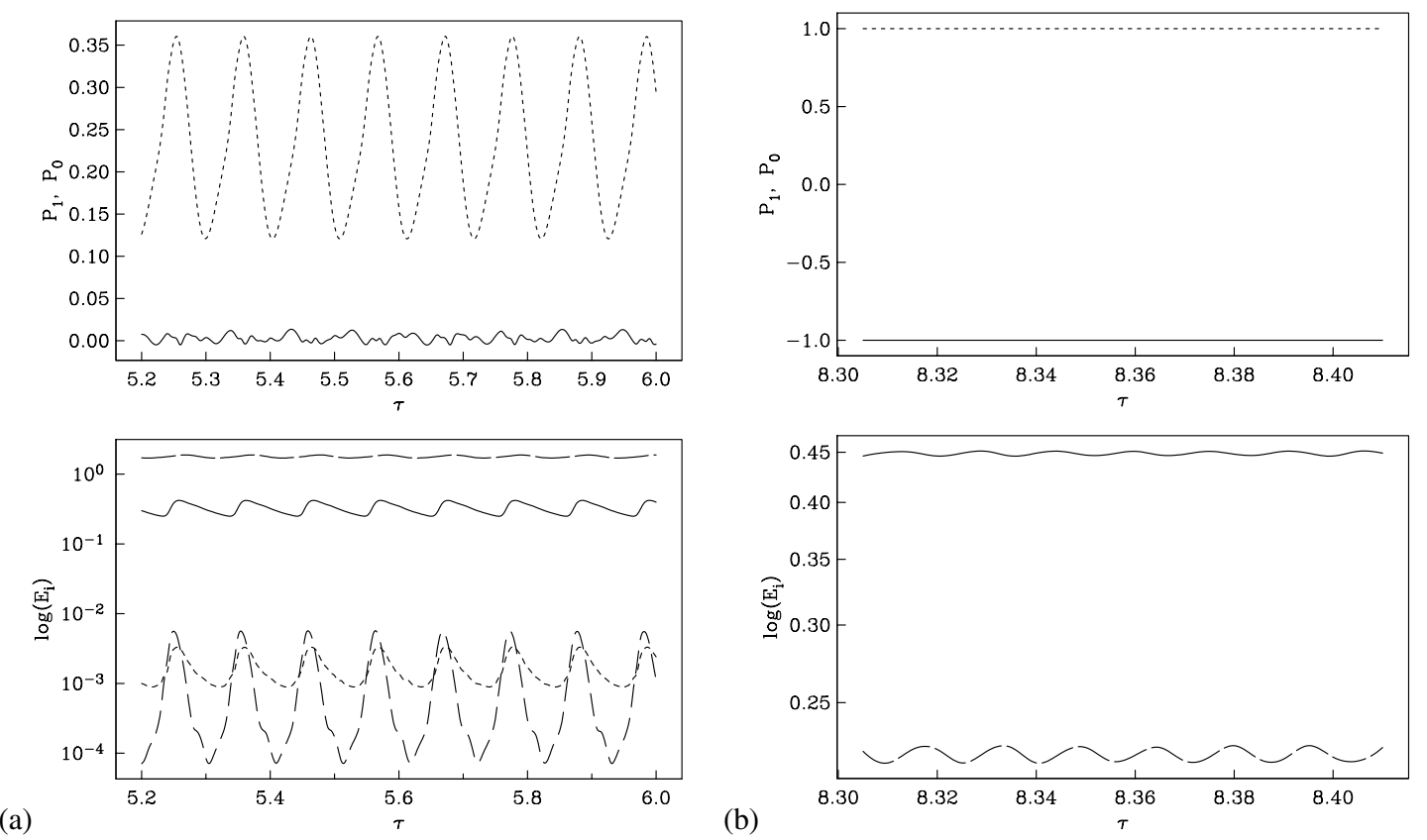

Fig. 5. For $r_{0}=0.77$, variations of parity (top - solid for $P_{1}$, broken for $P_{0}$ ) and modal energies (bottom - $E_{0}$ is upper long-dashed, $E_{1}$ is solid, $E_{2}$ is lower long-dashed, $E_{4}$ short-dashed) for $r_{0}=0.77, C_{\alpha}=-50$ and a) $\left.C_{\omega}^{*}=+500, \mathbf{b}\right) C_{\omega}^{*}=-500$. In b), only $E_{0}$ and $E_{1}$ are shown, to avoid too large vertical compression of the scale.
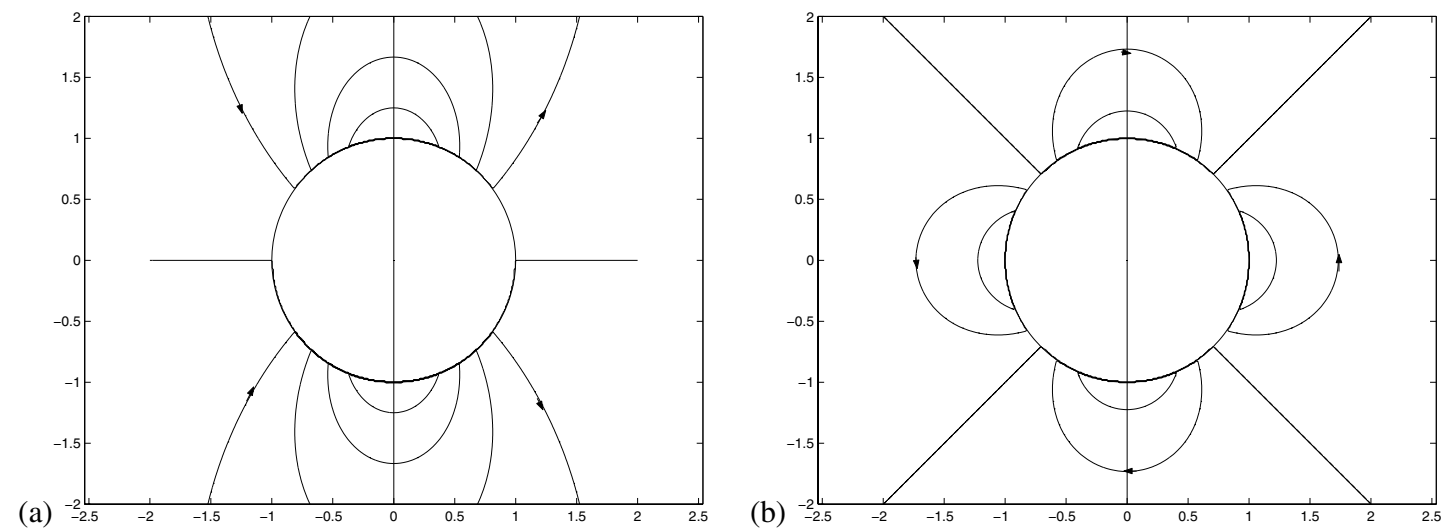

Fig. 6. Cross-sections of poloidal field lines for a) S1 field ("perpendicular dipole-like"); b) A1 field. The vertical line denotes the rotation axis.

field and an oscillating axisymmetric field. The "most active" longitude can then be plotted as a function of time.

We begin by looking at the solution for $r_{0}=0.64$, $C_{\alpha}=-50, C_{\omega}^{*}=-500$ (see Fig. 2b), in the final state with "pure" parities $P_{0}=P_{2}=+1, P_{1}=P_{3}=-1$. Figure 8 gives the space-time diagram of the latitudinally integrated surface field strength $\overline{B^{2}(\phi)}$. As a consequence of the strict equatorial symmetries of the solution, there are in general two welldefined maxima, separated by $180^{\circ}$. What is perhaps more surprising is that these maxima periodically jump in longitude, by about $-60^{\circ}$. In Fig. 8, such jumps can be seen at, e.g., $\tau \approx 7.38$ and 7.41 (remembering that $-60^{\circ}$ is here equivalent to $+120^{\circ}$ ). This results from the interaction of the travelling wave in the $m=0$ field, typically with maximum at mid-latitudes, with the $m \geq 1$ part of the field (predominantly in the $m=1$ component) which is concentrated at high latitudes. That the contours of the $m=1$ field are tilted (as hinted at in Fig. 2) accentuates the phenomenon. The smaller, but here not insignificant, $m>1$ components add some fine structure.

As mentioned earlier, what is seen will depend on the line of sight relative to the rotation axis. If, approximating a smaller inclination angle, attention is restricted to a single hemisphere, then there is a single longitude of maximum $\overline{B^{2}(\phi)}$ at a given time, rather than two. Figure 9 shows the evolution of the "active longitude" in the $\mathrm{S}$ hemisphere, as a function of time, in a frame rotating with angular velocity $\Omega_{0}$. Because of the limitation to one hemisphere, jumps are of about $120^{\circ}\left(=(180-60)^{\circ}\right)$. The other important factor is the magnitude of the contrast between active and other longitudes. Given the unknown nature of the physical mechanisms involved, this is hard to quantify, but in this example in a single hemisphere the latitudinally integrated surface field strength is $30 \%-40 \%$ larger at maximum than minimum. 


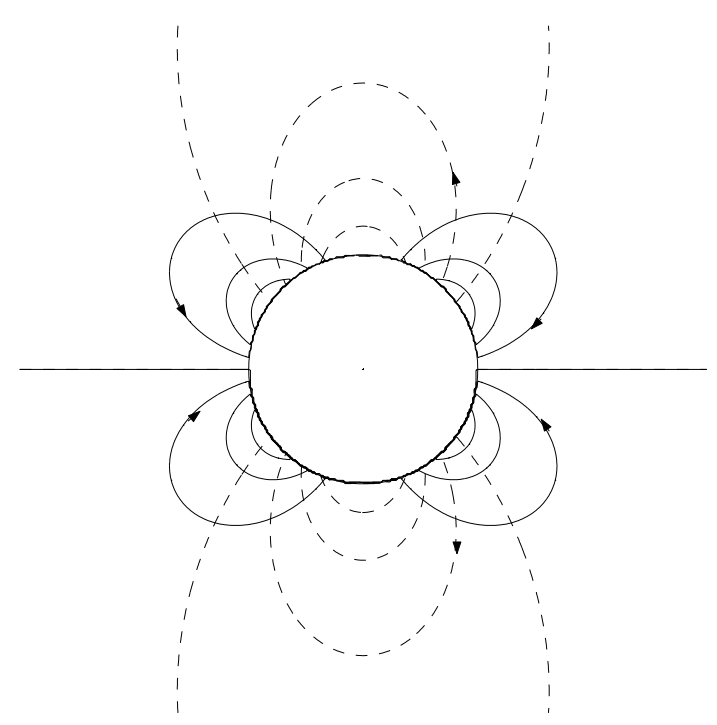

Fig. 7. A sketch of superimposed magnetic fields of S0-type (solid) and S1-type (broken). With the fields directed as indicated, the latitudinally averaged field has a maximum at longitude $0^{\circ}$ (on the right). When the direction of the S0 field is reversed, the maximum is at longitude $180^{\circ}$.

We now consider in a similar manner the model with $r_{0}=0.64, C_{\alpha}=-50, C_{\omega}^{*}=+500$ (as Fig. 2a). Now the nonaxisymmetric field is considerably weaker, and concentrated (in this case) to the southern hemisphere $\left(P_{1} \approx 0\right)$. To reveal the key features, we consider just the $m=0$ and $m=1$ parts of the solution in the southern hemisphere. Figure 10 is the spacetime diagram for the integrated squared surface field strength $\overline{B^{2}(\phi)}$. When comparing the series $\phi_{m}(t)$ with this figure, it is clear that there may be "false alarms", and we show instead in Fig. 12 (lower panel) the dependence of the latitude $\phi_{B}(t)$, the latitude of the maximum of the subsurface field strength. Jumps occur twice in each oscillation period of the $m=0$ part of the field. This procedure may not be as arbitrary as it appears - viewed from more nearly "overhead" ( smaller inclination angle) the high latitude features will contribute more to the observed signal. The upper panel shows the corresponding values of $B_{\max }^{2}$ as a function of time. Note that the behaviour in Fig. 10 $i$ periodic - features drift out of the top of the figure at longitude $360^{\circ}$ to remerge at the bottom at longitude $0^{\circ}$. Jumps in longitude in Fig. 12 are typically $160^{\circ}-180^{\circ}$. Although weak, these events arguably have some of the characteristics expected of a "flip-flop".

The models with $r_{0}=0.77$ tend to have relatively stronger nonaxisymmetric components. As $C_{\alpha}<0$ here, then consistent with the "parity selection rules" mentioned in Sect. 3.1, those with $C_{\omega}^{*}<0$ have pure parities $P_{0}=+1, P_{1}=-1$ whereas those for $C_{\omega}^{*}>0$ resemble the corresponding solution described above when $r_{0}=0.64$. Space-time diagrams for $C_{\alpha}=-50, C_{\omega}= \pm 500$ are shown in Fig. 11, cf. the corresponding Figs. 8 and 10 for $r_{0}=0.64$. However, in spite of these similarities, the relatively small variations in the dominant axisymmetric field $E_{0}$ (Fig. 5) mean that changes in active longitude are more marginal events, with one of the two strong longitudes having only a very slightly stronger measure of field strength than the other. Thus we do not present analogues of Figs. 9 and 12.

\subsection{Summary}

This analysis shows that jumps of active longitude, not necessarily by $180^{\circ}$, do occur in certain dynamo models. However the magnitudes of the difference between the strengths of the weaker and stronger longitudes are not necessarily very large in these models, even when the strengths of the $m=0$ and $m=1$ field components are comparable. This seems to be because the computed fields in the examples treated in detail do not have the idealized geometries described in Sect. 4.1. In particular, the relatively larger nonaxisymmetric fields occur when $C_{\omega}^{*}>0$, and the field configuration is least favourable. When $C_{\omega}^{*}<0$, the effects of the more favourable geometry are counterbalanced by the weaker nonaxisymmetric fields.

However the degree of arbitrariness in the procedures described in Sect. 4.2 to determine the active longitudes should be kept in mind - it is unclear which, if either, of $\phi_{m}$ and $\phi_{B}$ is the better measure, and it is not uncertain that all the "false alarms" mentioned really would be unobservable. Thus there is the possibility that with a realistically complex field structure flipflops occur more frequently than suggested by Figs. 9 and 12.

\section{Discussion}

The results presented above suggest that, in a limited range of parameters, distributed mean field dynamos are capable of generating significant nonaxisymmetric fields in stars with quasi-cylindrical rotation laws, such as are expected to be present in stars that rotate rather more rapidly than the Sun. Nonaxisymmetric fields are more readily produced in stars with relatively shallow dynamo-active regions ("convection zones") as in Sect. 3.3. For $C_{\alpha}<0$, anti-solar differential rotation (i.e. equatorial deceleration) appears to facilitate their generation. Thus active longitudes can be expected to occur quite widely. Nonaxisymmetric features are stronger at higher latitudes. Such a feature for axisymmetric fields was found by Bushby (2003), but Covas et al. (2005) show this to be a rather model dependent feature. The result for the nonaxisymmetric part of the field is also consistent with the idea that it is radial shear that destroys nonaxisymmetric field, and that with the rotation law (2) this shear is reduced at higher latitudes.

A topical question is whether any of these models display the "flip-flop" effect. Simple arguments show that a combination of an oscillating axisymmetric quadrupole-like field (S0) and a steady high latitude S1 field ("perpendicular dipolelike") will have longitudes of maximum field strength that alternate by $180^{\circ}$ every half period of the oscillating field (again see Moss 2004), and similar arguments can be presented for other combinations of oscillatory axisymmetric and quasisteady nonaxisymmetric fields (see also Fluri \& Berdyugina 2005). Clearly some intermediate configurations will also display a form of the phenomenon, although for anharmonic variations of both axisymmetric and nonaxisymmetric fields, jumps in general will not be of $180^{\circ}$, nor will they generally occur 


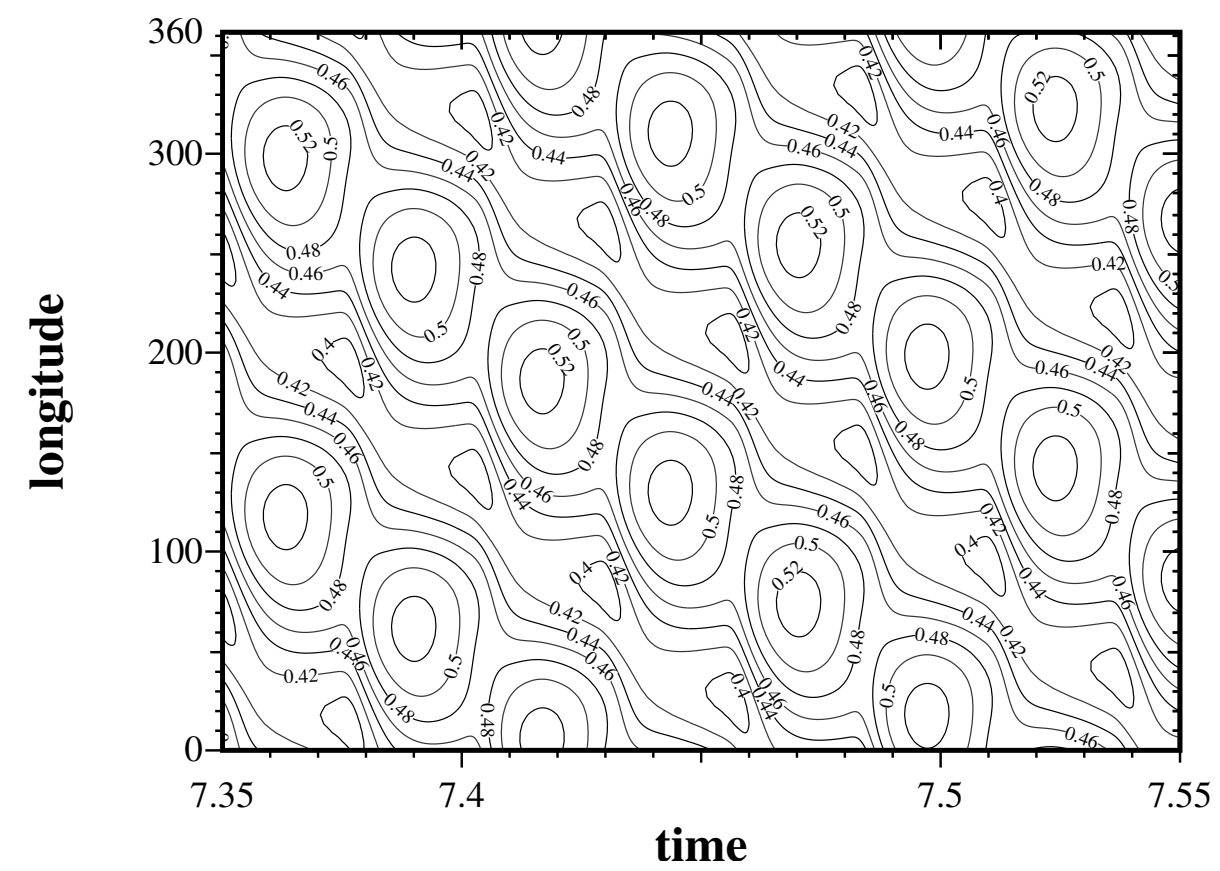

Fig. 8. Space-time diagram of longitudinally integrated surface field strength for the solution with $r_{0}=0.64, C_{\alpha}=-50, C_{\omega}^{*}=-500$.

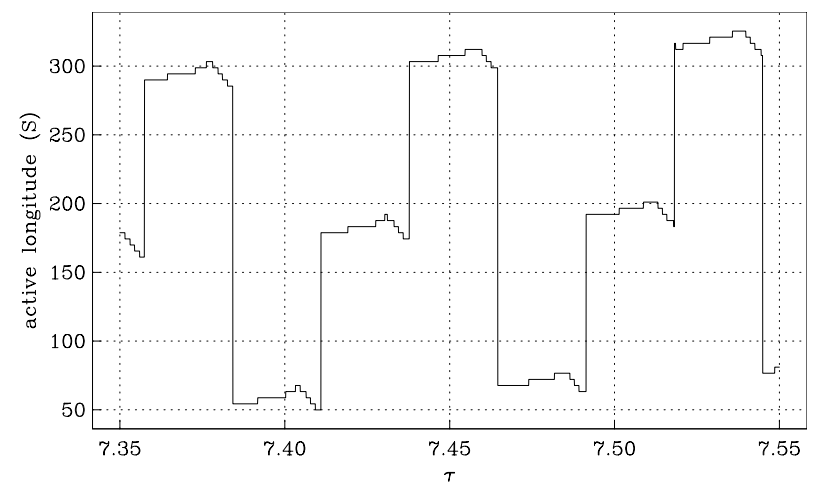

Fig. 9. The longitude of maximum integrated squared surface field strength as a function of time for the $\mathrm{N}$ hemisphere, $r_{0}=0.64$, $C_{\alpha}=-50, C_{\omega}^{*}=-500$.

with a periodicity of half the oscillation period of the axisymmetric field. In this context, an important uncertainty is the threshold of degree of nonaxisymmetry $\mathcal{M}$ above which flipflop behaviour will be apparent to an observer. (In fact, it is the near-surface field configuration that is plausibly relevant, rather than the global field but, as mentioned earlier, there is not a significant difference between global and near-surface values of $\mathcal{M}$.)

Models with $C_{\alpha} C_{\omega}^{*}<0$ (equatorial acceleration, if $C_{\alpha}<0$ ) possess weaker nonaxisymmetric fields: i.e. if we believe that this is the appropriate sign for $C_{\alpha}$, these are the rotation laws that appear to have the most observational support. The opposite sign of $C_{\alpha} C_{\omega}^{*}$ is more favourable for generation of nonaxisymmetric fields, but now flip-flops will only be observed from relatively small inclinations, so that one hemisphere is preferentially viewed. However field parities (for axisymmetric fields, at least) can be sensitive to quite small model details.
Thus, the possibility exists that relatively minor modifications could yield more "favourable" configurations.

Models discussed above have a simple alpha-quenching nonlinearity. Other forms of nonlinearity are possible, for example buoyancy and in particular the reaction of the azimuthal Lorentz force onto the rotation field (see, e.g., Moss et al. 1995; Moss \& Brooke 2000; Covas et al. 2005; and specifically in the solar context, Covas et al. 2004). It is conceivable that were such a nonlinearity to be effective, the rotation field might adjust to a configuration that more strongly supports nonaxisymmetric field generation - cf. also Moss et al. (1995). Another refinement that would be likely to favour nonaxisymmetric field generation is the inclusion of anisotropy of the alpha-tensor (e.g. Rüdiger \& Elstner 1994; Moss \& Brandenburg 1996).

The existence of steady purely nonaxisymmetric fields for certain parameter values is of at least theoretical interest. Such configurations were postulated to be the source of the nonaxisymmetric fields of the magnetic CP stars (Krause \& Oetken 1976). Here the dynamo was posited to operate within the convective core of the middle main sequence stars (where the rotation law is unknown), generating a field with angle $\chi=\pi / 2$ between magnetic and rotational axes. This theory suggested that the observationally derived angle $\chi \neq \pi / 2$ between the rotation and magnetic axes was an artefact of the techniques used to analyse the observations. Difficulties with the theory included the absence of dynamo models with the required properties. In principle, it seems that some of these specific difficulties might be met by the sort of dynamo models discussed here. But this would require purely nonaxisymmetric fields to be generated in entire spheres, and it appears that thin shells are more favourable. A priori, it cannot be ruled out that the "surface" boundary conditions (i.e. at the boundary of the convective core) might be sufficiently different as to change this 


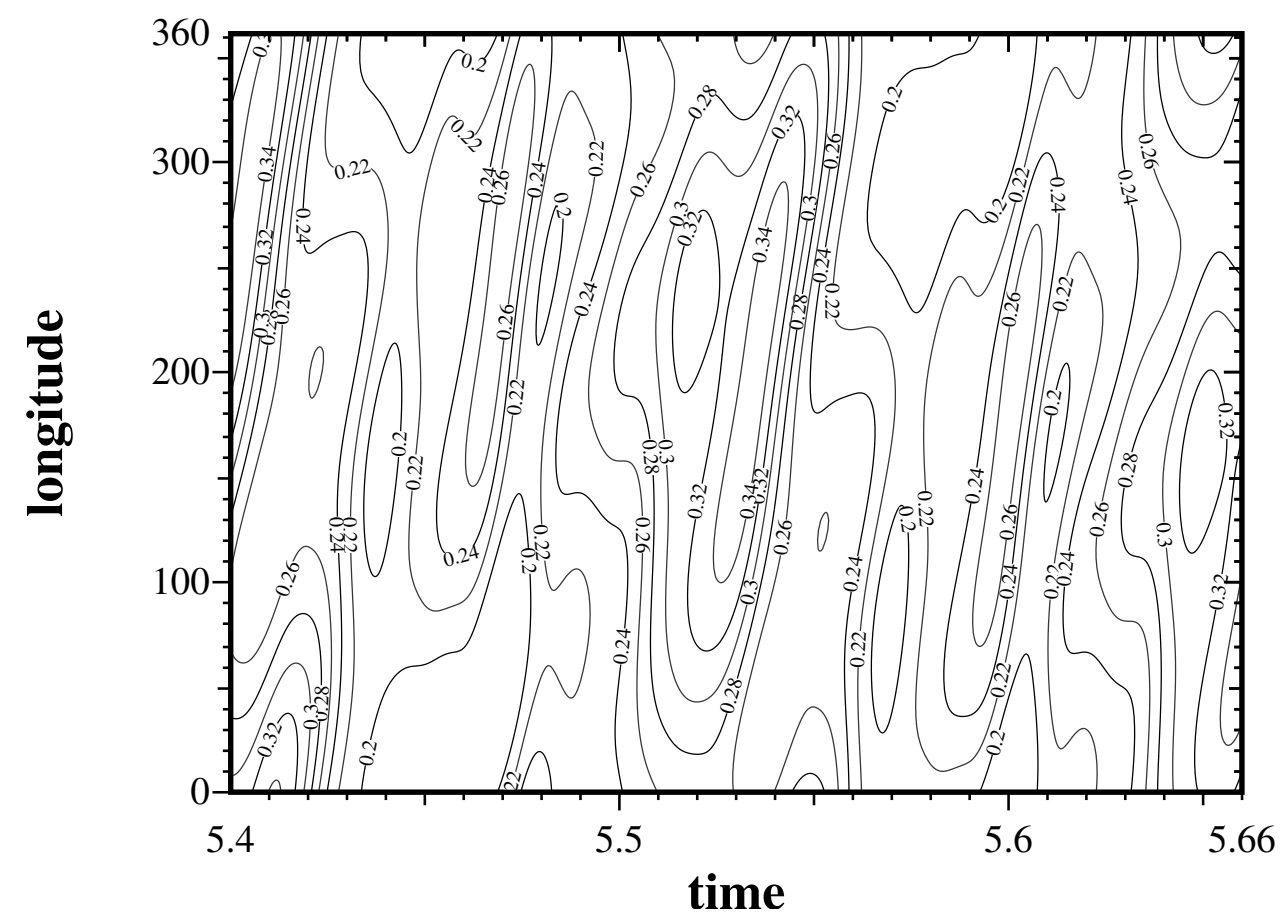

Fig. 10. Space-time diagram of longitudinally integrated squared surface field strength for the solution with $r_{0}=0.64, C_{\alpha}=-50, C_{\omega}^{*}=+500$.

(a)

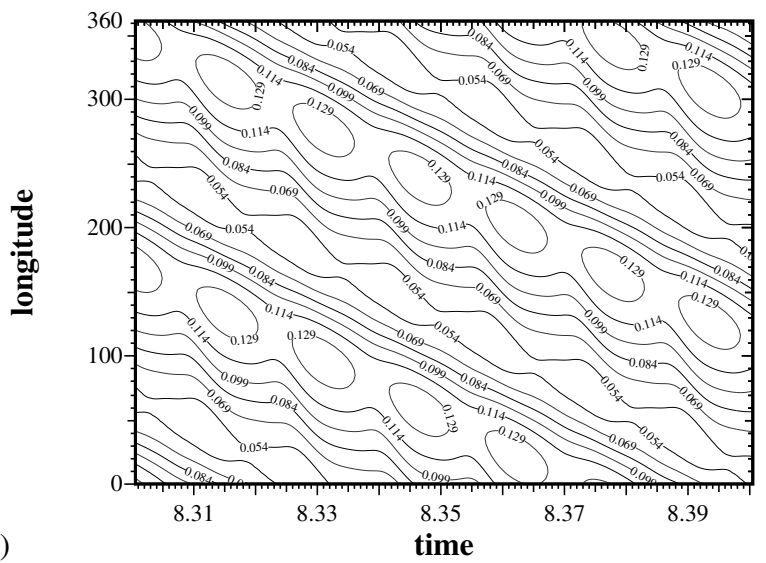

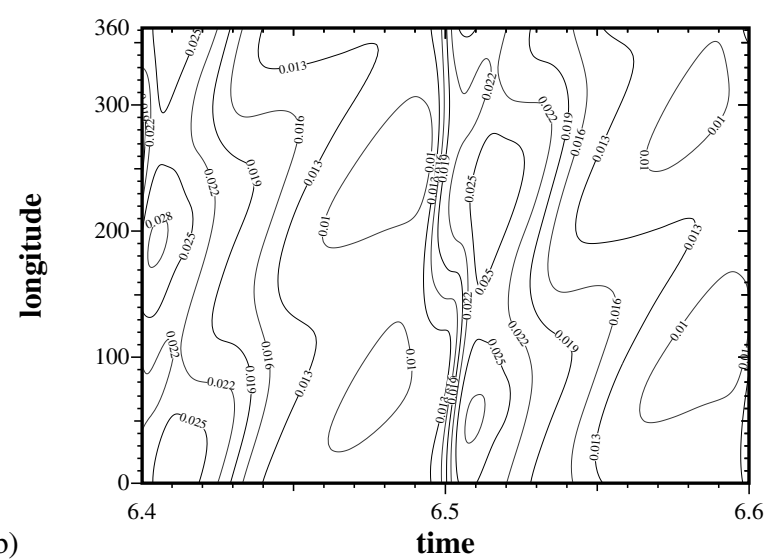

(b)

Fig. 11. Space-time diagrams of longitudinally integrated squared surface field strength for $r_{0}=0.77, C_{\alpha}=-50$ and a) $C_{\omega}=+500$; b) $C_{\omega}=-500(\mathrm{~N}$ hemisphere $)$.

trend, but this is perhaps improbable. In any case, other theoretical considerations and modern observations also throw severe doubt on this idea (see e.g. Moss 1989, 2001; Browning et al. 2004).

\section{Conclusions}

The most important result is that plausible rotation laws for rapidly rotating convective envelopes can support the generation of large-scale nonaxisymmetric magnetic fields, provided that the degree of differential rotation is not too large. Thus "active longitudes", associated with azimuthal maxima of suitable measures of surface field strength, can be expected to occur. In this context, there are suggestions that as rotation speeds increase, surface differential rotation in general tends to decrease (e.g. Hall 1991; Donahue 1993; Messina \& Guinan 2003; Reiners \& Schmitt 2003), and so the key parameter $C_{\omega}^{*}$ may vary much less widely than the mean rotation rate $\sim \Omega_{0}$. Some of the computed fields have the potential to exhibit flipflop behaviour but what is observable depends on a number of rather ill-determined factors. The simplest models predict that flip-flops will occur twice in a magnetic cycle (e.g. Moss 2004; and Sect. 4.2) but more complex field structures occur in the models discussed here (and on the surfaces of real stars!). In general, stable nonaxisymmetric fields are more readily generated in shallower dynamo active regions. This can be understood as follows: differential rotation preferentially reduces the length scale of nonaxisymmetric fields compared with axisymmetric, so enhancing nonaxisymmetric field decay. Shallower dynamo regions reduce the scales of both types of field, 

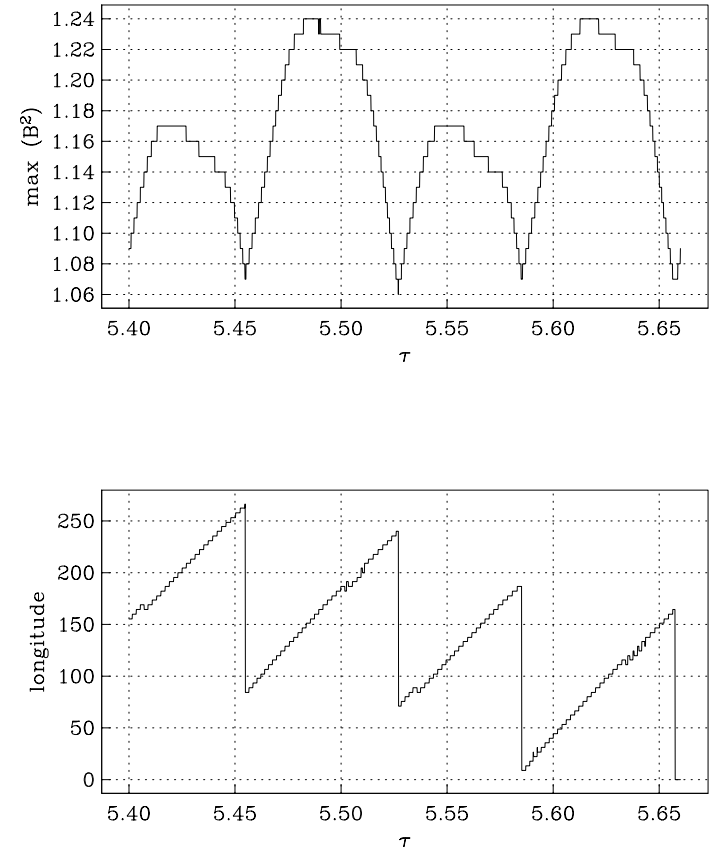

Fig. 12. The lower panel shows the longitude of maximum squared surface field as a function of time for the $\mathrm{S}$ hemisphere, $r_{0}=0.64$, $C_{\alpha}=-50, C_{\omega}^{*}=+500$; in the upper panel is the dependence of $B_{\max }^{2}$ on time. The small-scale "steps" are an artefact of the sampling.

reducing the relative discrimination against nonaxisymmetric fields; however the strength of any measure of active longitude is reduced in models with the thinnest dynamo-active shells.

Other points of interest include that, in general, "anti-solar" differential rotation tends to give rather stronger nonaxisymmetric fields (taking $C_{\alpha}<0$ ). Also, rather unusually, nonaxisymmetric fields are sometimes found to be oscillatory. This may simply be a consequence of the linking of axi- and nonaxisymmetric fields via the alpha-quenching nonlinearity - as the axisymmetric field oscillates, the strength of alpha-quenching changes, giving a modulation of the nonaxisymmetric field.

This study only begins to hint at the richness of behaviour connected with nonaxisymmetric field generation in stars with significant convective envelopes. Given the potential sensitivity of certain features of the models to unknown factors (such as boundary conditions, imposed spatial dependence of $\alpha_{0}$ and $\Omega$, etc.), and the corresponding freedom to modify significantly the model, further investigations may well produce other interesting results. Covas et al. (2005) have made a preliminary study of variations in differential rotation, arising from the action of the azimuthal part of the Lorentz force, in an otherwise quite similar model.

\section{References}

Barker, D. M., \& Moss, D. 1994, A\&A, 283, 1009

Bassom, A., Soward, A., Kuzanyan, K., \& Sokoloff, D. 2005, GAFD, submitted

Berdyugina, S. V., \& Tuominen, I. 1998, A\&A, 336, 25

Berdyugina, S. V., Pelt, J., \& Tuominen, I. 2002, A\&A, 394, 505

Berdyugina, S. V., \& Usoskin, I. G. 2003, A\&A, 405, 1121

Brandenburg, A., Krause, F., Meinel, R., Moss, D., \& Tuominen, I. 1989, A\&A, 213, 411

Browning, M. K., Brun, A. S., \& Toomre, J. 2004, in preparation

Bushby, P. 2003, MNRAS, 342, L15

Collier Cameron, A., \& Donati, J. F. 2002, MNRAS, 329, L23

Covas, E. O., Moss, D., \& Tavakol, R. 2004, A\&A, 416, 775

Covas, E. O., Moss, D., \& Tavakol, R. 2005, A\&A, 429, 657

Donahue, R. A. 1993, PASP, 105, 804

Donati, J.-P., Collier Cameron, A., \& Petit, P. 2003, MNRAS, 345, 1187

Fluri, D. M., \& Berdyugina, S. V. 2005, Sol. Phys., in press

Hall, D. S. 1991, in The Sun and Cool Stars: Activity, magnetism, dynamos, ed. I. Tuominen, D. Moss, \& G. Rüdiger (Heidelberg: Springer), Proc. IAU Coll., 130, 353

Henry, G. W., Eaton, J. A., Hamer, J., \& Hall, D. S. 1995, ApJS, 97, 513

Jardine, M., Collier Cameron Cameron, A., \& Donati, J.-F. 2002, MNRAS, 333, 339

Jetsu, L. 1996, A\&A, 314, 153

Jetsu, L., Pelt, J., Tuominen, I., \& Nations, H. L. 1991, in The Sun and Cool Stars: Activity, magnetism, dynamos, ed. I. Tuominen, D. Moss, \& G. Rüdiger (Heidelberg: Springer), Proc. IAU Coll., 130,381

Krause, F., \& Oetken, L. 1976, in Physics of Ap Stars, ed. W. W. Weiss, H. Jenkner, \& H. J. Wood, IAU Coll., 32, 29

Korhonen, H., Berdyugina, S. V., Strassmeier, K. G., \& Tuominen, I. 2001, A\&A, 379, L30

Korhonen, H., Berdyugina, S. V., \& Tuominen, I. 2002, A\&A, 390, 179

Messina, S., \& Guinan, E. F. 2003, A\&A, 409, 1017

Moss, D. 1989, MNRAS, 236, 629

Moss, D. 1999, MNRAS, 306, 300

Moss, D. 2001, in Magnetic fields across the HR diagram, ed. G. Mathys, S. K. Solanki, \& D. T. Wickramasinghe (San Francicsco: ASP), 305

Moss, D. 2004, MNRAS, 352, L17

Moss, D., Tuominen, I., \& Brandenburg, A. 1991, A\&A, 245, 129

Moss, D., Barker, D. M., Brandenburg, A., \& Tuominen, I. 1995, A\&A, 294, 155

Moss, D., \& Brandenburg, A. 1996, GAFD, 80, 229

Moss, D., \& Tuominen, I. 1997, A\&A, 321, 151

Moss, D., \& Brooke, J. M. 2000, MNRAS, 315, 521

Moss, D., Piskunov, N., \& Sokoloff, D. 2002, A\&A, 396, 885

Rädler, K.-H. 1986, in Plasma Astrophysics, ESA, SP-151, 569

Rädler, K.-H., Weidemann, E., Meinel, R., Brandenburg, A., \& Tuominen, I. 1990, A\&A, 239, 413

Reiners, A., \& Schmitt, J. H. M. M. 2003, A\&A, 398, 647

Rüdiger, G., \& Elstner, D. 1994, A\&A, 281, 46 